Meeting report

\title{
Tuberculosis 2012: biology, pathogenesis and intervention strategies; an update from the city of light
}

Available online 20 December 2012

\begin{abstract}
Tuberculosis (TB) remains one of the world's most deadly infectious diseases, with approximately 1.5 million deaths and 9 million new cases of TB in 2010. There is an urgent global need to develop new control tools, with advances necessary in our basic understanding of the pathogen, Mycobacterium tuberculosis, and translation of these findings to public health. It was in this context that the "Tuberculosis 2012: Biology, Pathogenesis, Intervention Strategies" meeting was held in the Institut Pasteur, Paris, France from 11 to 15th Sept 2012. The meeting brought together over 600 delegates from across the globe to hear updates on the latest research findings and how they are underpinning the development of novel vaccines, diagnostics, and drugs.
\end{abstract}

(C) 2012 Institut Pasteur. Published by Elsevier Masson SAS. All rights reserved.

Keywords: Mycobacteria; Tuberculosis; Virulence; Infectious diseases

\section{Introduction}

Paris has a long and distinguished history of international conferences dealing with tuberculosis (TB), dating back to 1867 and the first international conference of Internal Medicine when Jean-Antoine Villemin proposed that TB was a contagious disease (Enarson, 2004). The "Tuberculosis 2012: Biology, Pathogenesis, Intervention Strategies" meeting held at the Institut Pasteur, Paris, from 11 to 15 th Sept 2012 certainly lived up to this proud tradition, with an outstanding array of speakers, over 300 poster presentations, and over 600 delegates. It would be impossible in this short report to cover the breadth of science presented at the meeting in the detail it deserves, but we have tried herein to summarise some of the salient advances. We have structured our review along the same lines as the conference oral sessions, and would like to thank all our colleagues for their permission to present their unpublished work in this format.

\section{Opening session}

The opening session set the tone for the following days, mixing basic research with current progress in halting the TB epidemic. Hannu Laang (EC, Brussels) provided insight into the
European TB funding landscape, while Christian Lienhardt (WHO, Switzerland) gave an overview of progress in achieving the TB reduction targets set out by the Millennium Development Goals and the STOP TB Partnership. There are clearly some 'good news' stories, with the success of DOTs and the declining global TB incidence rates. However, there are still many problems facing us, including that one third of TB cases are not diagnosed or treated; the emergence of XDR and MDR TB; and the continuing synergy between HIV and TB in Africa. Clearly we are still some distance from a world without tuberculosis.

The role of basic science in providing new approaches to TB control was highlighted by two keynote lectures. Patrick Brennan (Colorado State University, Fort Collins, USA) brought us on a wonderful journey through his 50 years of research on TB and leprosy, highlighting how the work of his group and others has revealed the complexity and biosynthesis of the mycobacterial cell wall. Stewart Cole (EPFL, Lausanne, Switzerland) then presented a journey through TB time and space, weaving together the global dissemination of $\mathrm{Myco-}$ bacterium tuberculosis with its dissemination through the host, the latter process being controlled in part by the EspR nucleoid associated protein (NAP) (Blasco et al., 2012). The role of EspR in controlling expression of a number of virulence factors reveals the potential of such NAPs as novel drug targets. 


\section{Mycobacterial evolution, genetic diversity and conservation}

The application of genome sequencing to $M$. tuberculosis has led to enormous advances in our understanding of the evolution and global dissemination of M. tuberculosis. This theme was continued at the meeting, with new data presented by Roland Brosch (Institut Pasteur, Paris, France) on the genome sequences of smooth strains of Mycobacterium canetii (Brisse et al., 2006; Gutierrez et al., 2005; van Soolingen et al., 1997). Their analyses demonstrate that, in contrast to comparisons between other $M$. tuberculosis complex members, the smooth strains are genetically more diverse, with high numbers of SNPs and evidence for recombination. The geographical isolation of the smooth TB strains to East Africa may indicate that they are less adept at maintenance in human populations, suggesting that comparative analyses between smooth strains and other M. tuberculosis lineages may uncover key traits that led to the latter's global success.

While the relatively low genetic diversity of $M$. tuberculosis isolates is well described, this does not translate into phenotypic homogeneity, a fact emphasised by Stefan Niemann (Borstel Research Centre, Germany). Drawing on an international collection of M. tuberculosis lineages, they have shown that there are distinct differences in these strains' transcriptomes and interaction with macrophages (Homolka et al., 2010). These lineage specific traits may reflect the evolution of $M$. tuberculosis with its human host, a theme developed by Iñaki Comas (Centre for Public Health Research, Valencia, Spain) who proposed a model for co-evolution between $M$. tuberculosis and humans. He put forward the intriguing suggestion that the emergence of 'modern', highly virulent $M$. tuberculosis is a consequence of increases in human population density over the last 70,000 years, with low virulence $M$. tuberculosis having predominated until the last 10,000 years.

\section{Mycobacterial biology and physiology}

The mycobacterial cell wall has long been known to have an array of complex lipids, but defining their exact roles has remained elusive. Christophe Guilhot (IPBS, Toulouse, France) gave an overview of the roles of polyketide-derived lipids in TB biology. He showed evidence for synergistic interactions between lipids such as acyltrehaloses and sulfolipids in virulence, which may explain previous work showing that individual gene knock-outs in the respective biosynthetic pathways did not produce clear attenuation phenotypes in mouse models. The role of polyketide-derived lipids was also addressed by Rajesh Gokahle (CSIR-IGIB, Dehli, India), who is exploring the role of such lipids in mycobacterial biofilm formation and reported that Mycobacterium smegmatis pks mutants make defective biofilms; whether the same holds true for M. tuberculosis pks remains to be determined. Regulation of FasI and FasII systems was discussed by Gabriela Gago (IBR, Roasario, Argentina), who described the FasR and MabR regulators of the FasI and FasII systems, respectively (Arabolaza et al., 2010; Salzman et al., 2010).
The application of systems biology approaches to $M$. tuberculosis, with precise, quantifiable data on the abundance of transcripts, proteins, and metabolites, is set to provide an exquisite level of detail on the inner workings of $M$. tuberculosis. Olga Schubert (ETH, Zurich, Switzerland), elegantly demonstrated the power of such systems approaches in her presentation of the ' $M$. tuberculosis Proteome Atlas'. The proteome analysis was based on the identification of unique, 'proteotypic' peptides for every $M$. tuberculosis protein that were then used to develop selective reaction monitoring (SRM) assays (Picotti and Aebersold, 2012). This SRM approach opens the way for the precise quantification of $M$. tuberculosis proteins in a complex mixture, such as $M$. tuberculosis grown in a macrophage, and will provide us with valuable information on the relationship between transcript and protein abundance, protein turnover, etc.

In a meeting that was kicked-off by Patrick Brennan, it was apt that the name of Joseph Bigger cropped up during the meeting, Bigger having been the Professor of Bacteriology and Preventive Medicine in Patrick's alma mater, Trinity College Dublin, Ireland. Bigger's seminal work on bacterial persisters (Bigger, 1944) was placed in a modern context by John McKinney (EPFL, Lausanne, Switzerland) who presented the use of microfluidics and time-lapse microscopy to monitor the response of single bacterial cells to antibiotic treatment. Using this system they have uncovered a host of novel findings, including how pulsatile expression of $\mathrm{katG}$ activates isoniazid and hence killing of $M$. smegmatis, showing how stochastic gene expression across a population is one mechanism that generates variation in bacterial drug susceptibility.

\section{Novel transport systems in mycobacteria}

The disclosure of an outer membrane, the mycomembrane, in the mycobacterial cell envelope revealed another layer of complexity to the transport of products into, and out of, the cell (Hoffmann et al., 2008). The novel Type VII secretion system provides some answers as to how M. tuberculosis secretes proteins across this hydrophobic barrier, with $M$. tuberculosis possessing 5 such systems named ESX1-5 (Bitter et al., 2009). However, much work remains to be done to elucidate the exact workings of the ESX mechanism. Daria Bottai (University of Pisa, Italy) has focussed on the ESX-5 system and its roles in secretion of effector proteins from $M$. tuberculosis. Strikingly, the ESX-5 system was shown to be responsible for secretion of a large number of PE and PPE proteins, with loss of the ESX-5 channel protein, EccD5, blocking secretion (Bottai et al., 2012). The ESX-5 secreted $\mathrm{PE} / \mathrm{PPE}$ proteins were shown to be important immunogens underlining the key role of the ESX-5 system in the host's immune response to $M$. tuberculosis (Sayes et al., 2012). The ESX-5 secretion system was further dissected by Edith Houben (VU Medical Centre, Amsterdam, The Netherlands), who showed that the ESX-5 membrane channel is a multiprotein complex made up of EccBCDE (Houben et al., 2012a,b). The exact configuration of these components is still unclear, but 
EccE may form the outer pore and hence enable the EccBCDE complex to generate an integrated pore across both the inner membrane and mycomembrane.

The problem of getting nutrients into M. tuberculosis was addressed by Alexandre Gouzy (IPBS Toulouse, France) who dealt with nitrogen acquisition. Focussing on the AnsP1 and AnsP2 transporters, which were both originally annotated as putative asparagine transporters, he used a combination of mutagenesis and biochemical characterisation to reveal them to in fact be aspartate (AnsP1) and asparagine (AnsP2) transporters. He then explored the roles of these transporters in vivo, and presented compelling evidence that AnsP1 is required during the early stages of infection in mice.

Michael Niederweis (University of Alamaba, Birmingham, USA) concluded this session by discussing the search for porins in M. tuberculosis (Niederweis et al., 2010). He presented an intriguing story on the identification of a novel outer membrane channel, MptA, in Mycobacterium bovis BCG and M. tuberculosis. As well as its role in solute transport, the results suggested this protein may also function as a secreted toxin, calling to mind secreted autotransporter toxins in Enterobacteriaceae (Guyer et al., 2000).

\section{Adaptation to changing environment}

A large part of the success of M. tuberculosis as a major pathogen relies on its ability to adapt to a variety of environments, to switch its metabolic needs from one source to another, and to resist a number of toxic conditions encountered during infection. A whole session of the meeting was dedicated to mycobacterial adaptation to changing environments, taking examples from processes involved in gene regulation, nutrient assimilation and resistance to host immunity and antibiotic treatment.

The adaptation process in M. tuberculosis requires fast and subtly controlled mechanisms to regulate gene expression. Kristine Arnvig (NIMR, London, UK) described the contribution of non-coding RNAs to shaping the M. tuberculosis transcriptional landscape (Arnvig and Young, 2009, 2012; Arnvig et al., 2011). Using RNA sequencing, she showed that small non-coding RNAs are differentially expressed by $M$. tuberculosis in various environments, such as in in vitro culture, macrophages or mice. Nearly a fifth of the RNA sequencing reads map to intergenic regions in M. tuberculosis, a fraction that reaches over $50 \%$ in stationary phase conditions as a result of the accumulation of a highly abundant noncoding RNA, MTS2823. A potential role for these regulatory elements in $M$. tuberculosis adaptation to stress and virulence was suggested, as has been reported in many bacterial pathogens (Toledo-Arana et al., 2007); however, the exact targets and signals involved in non-coding RNA-mediated gene regulation in $M$. tuberculosis still remain to be understood. In addition to genetically encoded modulation of gene expression, Babak Javid (Tsinghua University, Beijing, China) proposed that random mistranslation might also be involved in $M$. tuberculosis physiology and resistance to antibiotics. In particular the involvement of this 'error generating process' in phenotypic resistance to rifampicin was reported.

Nutritional aspects in M. tuberculosis infection were explored from the viewpoint of vitamin capture and utilization. Digby Warner (University of Cape Town, South Africa) studied the role of the co-factor vitamin $\mathrm{B}_{12}$ in the physiopathology of M. tuberculosis. Screening of a wholegenome insertional mutant library allowed the identification of an ABC-type transport protein that is essential for vitamin $\mathrm{B}_{12}$ import in vitro. Interestingly, while $M$. tuberculosis is predicted to encode a complete pathway for de novo vitamin $\mathrm{B}_{12}$ biosynthesis, a previous study had shown that a mutant in the $\mathrm{B}_{12}$ transporter was attenuated in mice during the chronic phase of infection. Further research is required in order to establish a causal link between the observed phenotype and a defect in vitamin $B_{12}$ uptake, however these results suggest the potential importance of specific nutrient acquisition systems in M. tuberculosis virulence.

In addition to nutrient scavenging, M. tuberculosis has to resist hostile environments generated by the host immune system. At the same time, the immune system must balance the need for bacterial control with avoidance of inflammatoryinduced immunopathology. Illustrating this point, Christopher Sassetti (Univ. Massachusetts, Worcester, USA) elaborated on the role of nitric oxide (NO) production by macrophages during the course of $M$. tuberculosis infection. While the production of $\mathrm{NO}$ upon cell activation by IFN $-\gamma$ is required to kill intracellular bacteria, he disclosed that NO plays also a critical role in controlling inflammation, and in particular in inhibiting both activation and IL1 $\beta$ maturation, and early neutrophil recruitment to the site of infection (Mishra et al., 2013). This suggests that, unexpectedly, IFN- $\gamma$ may have a role in the control of inflammation through NO production, in addition to its well-known function in activating the immune system.

\section{The search for novel antigens and correlates of protection}

The development of knowledge-based and adapted intervention strategies, including prognostic tools, new drugs and more effective vaccines, to combat TB would benefit considerably from improvements in our understanding of latent infection. To begin the session, Camille Locht (INSERM, Institut Pasteur de Lille, France) summarized his work on the heparin-binding haemagglutinin (HBHA). Disruption of the M. tuberculosis hbha gene significantly affects mycobacterial interaction with epithelial cells and impairs extrapulmonary dissemination of M. tuberculosis in the mouse (Pethe et al., 2001). HBHA is also a powerful latency antigen, and induces high levels of IFN- $\gamma$ secretion by the peripheral blood lymphocytes of latently infected subjects (Hougardy et al., 2007). In TB patients, HBHA-specific IFN- $\gamma$ responses are very low, probably due to the induction of HBHA-specific regulatory $\mathrm{T}$ cells during active $\mathrm{TB}$; nevertheless, the response increases after chemotherapy. IFN- $\gamma$ responses to HBHA may thus contribute to protection against the disease. 
An approach using a combination of BCG and HBHA as a booster for teenagers and adults or as a post-exposure vaccine was then discussed (Corbiere et al., 2012).

Cecilia Lindestam Arlehamn (La Jolla Institute for Allergy and Immunology, USA) presented results of a genome-wide screen for HLA class II epitopes from M. tuberculosis, where $74 \%$ of the identified antigens had not been previously described as sources of CD4 T cell epitopes. The antigens identified were derived from almost every protein category, but with an overrepresentation of PE/PPE and Esx proteins. Using PBMC from human donors latently infected with $M$. tuberculosis, she went on to show that the response to $M$. tuberculosis is highly heterogeneous and confined to the CXCR3+CCR6+ memory Th1 cell subset.

Latently infected individuals constitute a hidden disease reservoir, and an improved knowledge of key host and pathogen factors controlling TB latency would greatly benefit TB control efforts. Gilla Kaplan (University of New Jersey, Newark, USA) emphasised the utility of the rabbit as a model of TB latency, using two M. tuberculosis strains to illustrate the host-pathogen dynamic. Rabbits infected with the $M$. tuberculosis CDC1551 strain show limited pathology, temporal decreases in TNF production, and control but not eradication of the pathogen. The bacilli can be reactivated following immune suppression, underlining the generation of a state of latency. On the contrary, rabbits infected with the $M$. tuberculosis HN878 strain develop an abundant fibrosis with extensive tissue damage driven by sustained TNF production. This model therefore offers a route to defining key determinants of protective immunity and latency (Subbian et al., 2012).

Biomarkers could facilitate rapid diagnosis and treatment of TB patients and allow preventive measures for latently infected individuals. Stefan Kaufmann (MPIIB, Berlin, Germany) reported on whole-blood gene expression profiling of pulmonary TB against other chronic inflammatory diseases such as sarcoidosis (Maertzdorf et al., 2012). This elegantly displayed the power of such global profiling approaches to define novel diagnostic biosignatures of TB infection. He then went on to discuss the miRNA components of the transcript signature that distinguishes TB from latent infection. In particular, miRNA-223 (a negative modulator of neutrophils) was differentially expressed during active TB, with miRNA-223 knockout mice highly susceptible to $M$. tuberculosis infection (A. Dorhoi et al., submitted).

\section{Novel vaccine strategies}

BCG confers variable protection against pulmonary $\mathrm{TB}$ in adults, demanding that new vaccines with better protection are developed. Carlos Martin (University of Zaragoza, Spain) gave an update on the development of a live attenuated vaccine based on the inactivation of two $M$. tuberculosis genes: $p h o P$, a transcriptional regulator of key virulence factors in $M$. tuberculosis, and fadD26 involved in fatty acid metabolism (Nambiar et al., 2012). This vaccine, called MTBVAC, has been demonstrated to be safe and protective in animal models and is now progressing to Phase I clinical studies. Leander Grode (Vakzine Projekt Management, Hannover, Germany) reported the results from the Phase I clinical trials of VPM1002, a urease-deficient recombinant BCG secreting listeriolysin (Grode et al., 2005). No serious adverse reactions have been observed, and characterisation of the immune response showed induction of greater polyfunctional T-cells and IFN- $\gamma$ production in those vaccinated with VPM1002 compared to those receiving BCG. While the latter approach used BCG expressing listeriolysin, Yuelan Yin (Yangzhou University, Yangzhou, China) took the converse approach with Listeria monocytogenes expressing an Ag85B-ESAT-6 fusion protein. Subcutaneous, intraperitoneal and intravenous vaccination routes with this recombinant Listeria were shown to generate specific Th1-type cellular immunity.

Peter Andersen (Statens Serum Institut, Copenhagen, Denmark) explored a different strategy to enhance BCG efficacy. The H56 subunit vaccine is composed of early, constitutive and late $M$. tuberculosis antigens, namely Ag85b, ESAT-6 and Rv2660c (Aagaard et al., 2011). Boosting BCG with $\mathrm{H} 56$ resulted in efficient containment of M. tuberculosis infection in macaques and prevented reactivation of latent infection (Lin et al., 2012a,b). Helen McShane (University of Oxford, UK) finished this session with another strategy to boost BCG. She reported on the Phase I/IIa studies with the candidate TB vaccine Modified Vaccinia virus Ankara expressing Antigen 85A (MVA85A) from M. tuberculosis in healthy volunteers previously vaccinated with BCG (Pathan et al., 2012; Scriba et al., 2012). Two phase IIb trials in BCG-vaccinated infants and in HIV + adults are currently ongoing.

\section{Biochemistry and chemical biology I and II}

Adaptation of M. tuberculosis to its environment, and most particularly to the phagosomal compartment of the macrophage, is an essential component of its pathogenesis, transmission, and maintenance in the host (Schnappinger et al., 2003). Post-translational modifications, such as reversible acetylation or phosphorylation, are emerging as an important mechanism used by mycobacteria to adjust to environmental changes. John Blanchard (Albert Einstein College of Medicine, New-York, USA) reported that reversible acetylation by a cAMP-dependant protein lysine acetyltransferase reduces the activity of enzymes such as the central metabolic enzyme acetyl-CoA synthase (Xu et al., 2011); MbtA, an adenylating enzyme that catalyses the first step in the biosynthesis of the mycobactins; or FadD26 involved in phthiocerol dimycocerosate synthesis. Marco Bellinzoni (Institut Pasteur, Paris, France) detailed how the PknG substrate GarA, an FHAdomain protein, acts as a switch in central metabolism. Using combined structural and biochemical assays, it was shown how unphosphorylated/phosphorylated GarA controls the activity of a pivotal set of core enzymes, $\alpha$ ketoglutarate dehydrogenase, glutamate dehydrogenase and glutamate synthase, to direct metabolic flux towards the accumulation of glutamate (O’Hare et al., 2008; Wagner et al., 2011). 
Laurent Kremer (CNRS, Université de Montpellier, France) highlighted the role of Ser/Thr phosphorylation in the regulation of cell envelope biosynthesis by modulating the activity of enzymes involved in mycolic acid, peptidoglycan or glucan synthesis; of the MmpL7 phthiocerol dimycocerosate transporter; and of the transcriptional repressor EmbR that regulates the expression of arabinosyltransferases involved in arabinogalactan and lipoarabinomannan synthesis (Molle and Kremer, 2010). However, a number of questions still remain, such as the precise environmental signals that are sensed by acetylation/deacetylation and phosphorylation/dephosphorylation systems, if these systems are coordinated, and what physiological advantages are gained by these posttranslational modifications.

Functional assignment of all enzymes encoded by the genome of $M$. tuberculosis, and thus an understanding of its complete metabolic capacity, is far from complete. However Luiz Pedro de Carvalho (NIMR, London, UK) is endeavouring to fill this gap in our knowledge by using activity-based metabolomic profiling to permit unbiased discovery of enzymatic activities encoded by genes of unknown function. This approach applies liquid-chromatography mass spectrometry (LC-MS) to analyse the impact of a recombinant enzyme using a cellular extract as a physiological library of potential substrates and products (de Carvalho et al., 2010). In this way, the orphan enzyme encoded by Rv1692 was assigned as a Dglycerol 3-phosphate phosphatase. Emilie Layre (Brigham and Women's Hospital, Boston, USA) used a recently developed lipidomic methodology consisting of LC-MS analysis and bioinformatic tools for the broad and unbiased comparative analysis of M. tuberculosis total lipid extracts (Layre et al., 2011) to gain insight into the function of the type-III polyketide synthase Pks10. When comparing wild-type, deleted or over-expressing $M$. tuberculosis strains, she identified a new family of branched chain lipids produced by the $p k s 10$ overexpressing strain only, the precise structure of which is currently under investigation.

The rise in antibiotic-resistant M. tuberculosis emphasizes the need for new anti-TB agents with novel mechanisms of action. The meeting was very exciting in this aspect with an impressive amount of new anti-TB compounds and targets identified. It is worth noting that most of these in vitro or in vivo active compounds were obtained by screening chemical libraries on whole M. tuberculosis cells, in either replicating or non-replicating stages or inside phagocytes. As a perfect example of the strategies used, Carl Nathan (Weill Cornell Medical College, New York, USA) gave an overview of the first results obtained in a systematic whole-cell screen for compounds that kill non-replicating M. tuberculosis, replicating M. tuberculosis, or both (Gold et al., 2012). Over 2.5 million compounds provided by academic and industrial partners are due to be tested through funding from the Bill and Melinda Gates Foundation TB Drug Accelerator programme. Confirmed results from 113,000 compounds revealed that $0.5 \%$ of the compounds tested killed replicating $M$. tuberculosis selectively, $0.1 \%$ killed non-replicating $M$. tuberculosis selectively, and $0.03 \%$ killed both. Among compounds that showed bactericidal activity against $M$. tuberculosis, no activity against other bacteria and fungi, and limited toxicity to human hepatoma cells, were certain synthetic analogues of phytopyranocoumarins (calanolides) synthesized by P. Zheng and G. Liu of Tsinghua University. These latter compounds were found to be equipotent against replicating and nonreplicating bacilli, had a bactericidal mode of action, and killed bacilli residing in human macrophages. Their target awaits identification.

\section{Mycobacterial resistance and the need for new drug targets-search for novel drug candidates to cure TB}

Erik Böttger (University of Zurich, Switzerland) opened this session with a discussion of drug resistance in M. tuberculosis, from the viewpoints of clinical implications and fitness costs. The significant heterogeneity of $M$. tuberculosis drug resistance was one of the key messages of this presentation, i.e. low, medium and high level antibiotic resistance. The case was made for quantitative measures of drug resistance to complement the standard critical concentration methods that are currently used to define resistance (Bottger, 2011). Indeed, the mechanism of action for many TB drugs remains to be clarified, and it is surprising to think that the precise mechanism of action of pyrazinamide (PZA), introduced 60 years ago and one of the cornerstones of modern TB drug therapy, remains to be defined. Ying Zhang (Johns Hopkins University, Baltimore, USA \& Fudan University, Shanghai, China) reported the recent identification of RpsA as a novel target for PZA. RpsA acts in concert with tmRNA in trans-translation, a mechanism for releasing partially synthesized proteins from stalled ribosomes, a process of central importance to bacterial persisters; pyrazinoic acid binds to RpfA, blocking its ability to function in trans-translation (Shi et al., 2011).

Priscille Brodin (INSERM, Institut Pasteur de Lille, France) highlighted the potency of high-content cell-based screening and unbiased large scale phenotypic assays for the identification of novel host and mycobacterial genes involved in M. tuberculosis intracellular colonization (Fig. 1), as well as chemicals able to prevent bacterial intracellular growth (Christophe et al., 2010). During one of these screens in infected macrophages, ImidazoPyridine Amides (IPAs) were identified as potent drugs active against M. tuberculosis. As shown by Ulf Nehrbass (Institut Pasteur Korea, Seongnam, Republic of Korea), optimized IPAs inhibit the growth of MDR and XDR clinical isolates in the low nanomolar range. IPA Q203, despite being static in vitro, is cidal in the mouse model of M. tuberculosis infection, where it accumulates in the lung tissues and inhibits granuloma formation.

The pathway leading to the biosynthesis of mycolic acids is for the most part unique to mycobacteria and the utility of this as a drug target is clearly demonstrated by the therapeutic efficacy of several key anti-TB agents, such as isoniazid and ethionamide, as well as various other compounds, including thiolactomycin, isoxyl and thiacetazone. The primary mode of action of the latter two compounds was unknown, but Mary 


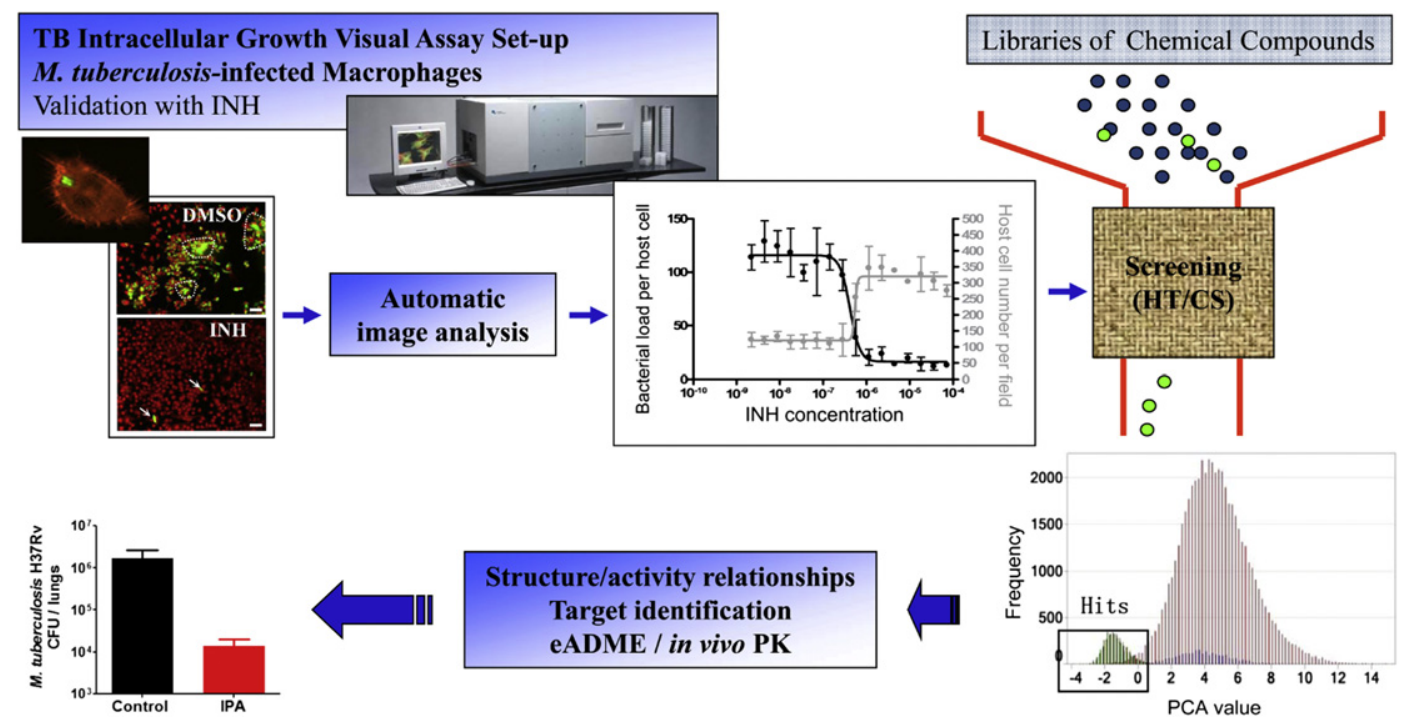

Fig. 1. A general approach for the discovery of novel anti-TB drugs based on high throughput/content screening on M. tuberculosis-infected macrophages. High throughput screening of chemicals that interfere with the replication of M. tuberculosis within macrophages is performed thanks to a phenotypic cell-based assay that uses automated confocal fluorescence microscopy. RAW macrophage cells are infected with M. tuberculosis H37Rv expressing GFP (pictures are shown at day 5 after infection). Chemical compounds are first screened at a single concentration; isoniazid (INH) is used as a control. A customized image analysis automatically quantifies several parameters such as the number of host macrophages, the percentage of infected cells, or the average surface area of bacterial aggregates that are then processed in a Primary Component Analysis (PCA). Hits are confirmed by means of serial dilutions, and MICs are determined. Leads are optimized in terms of efficacy, eADME and in vivo PK and the target is looked for before efficacy in mouse model of infection is being assessed by CFU reduction in lungs. The example of an IPA derivative developed by Institut Pasteur Korea and INSERM is given, covered by patents No et al. 18/03/2011, WO2011/113606 A1, PCT/EP 2011/001345. Figure courtesy of Priscille Brodin (INSERM, Institut Pasteur de Lille, France).

Jackson (Colorado State University, Fort Collins, USA) described the discovery of a common mechanism of action, i.e. inhibition of the dehydratase step of the Fatty Acid Synthase type II (FAS-II) elongation cycle, catalysed by the (3R)hydroxyacyl-ACP dehydratases encoded by hadABC (Grzegorzewicz et al., 2012a,b). Moreover, she reported the identification, thanks to a whole-cell M. tuberculosis screen, of an adamantyl urea compound that shows potent bactericidal activity in vitro and that had a unique mode of action, namely the abolition of the MmpL3 translocation of mycolic acids from the cytoplasm, where they are synthesized, to the periplasmic side of the plasma membrane (Grzegorzewicz et al., 2012a,b). Three additional independent studies have recently validated MmpL3 as a new and promising target, reporting compounds that target MmpL3 and inhibit M. tuberculosis growth (La Rosa et al., 2012; Stanley et al., 2012; Tahlan et al., 2012).

Benzothiazinones are highly active compounds that were previously shown to target the enzyme decaprenylphosphoryl$\beta$-D-ribose 2 -epimerase and kill M. tuberculosis by blocking the synthesis of the arabinan domain of arabinogalactan and lipoarabinomannan (Makarov et al., 2009). Based on the study of the metabolism, pharmacokinetics and mechanisms of resistance to two advanced compounds, Vadim Makarov (Bakh Institute of Biochemistry, Moscow, Russia) reported the design and synthesis by classical approaches of medicinal chemistry of a second generation of benzothiazinone derivatives with improved activity and bioavailability in mice.

Although phenotypic-based approaches provided the most spectacular results, three speakers demonstrated promising target-based strategies to identify new anti-TB drugs. Helena
Boshoff (NIH, Bethesda, USA) targeted the non-redundant fumarase enzyme acting in the reverse branch of the TCA cycle as they recently found that fermentation might play an important role in the maintenance of $M$. tuberculosis under the low oxygen tensions encountered in granulomas (Watanabe et al., 2011). In silico screening provided sulphonamides as lead compounds, inhibiting the recombinant enzyme and showing activity against anaerobic bacilli, that although moderate, validates the approach. Michael Zimmermann (ETH, Zurich, Switzerland) demonstrated that fermentative activity seems to be a common mycobacterial feature under anaerobia, but that the usage of metabolic pathways differs significantly between fast- and slow-growing mycobacteria.

Owing to the challenge in identifying and validating new targets, David Sherman (Seattle Biomedical Research Institute, USA) reconsidered targets/pathways validated in other organisms but under-exploited in TB chemotherapy, and gave the example of the folate pathway (Kumar et al., 2012). He described several compounds that inhibit M. tuberculosis dihydrofolate reductase and that synergize to kill the bacilli by depleting them of methionine derivatives. As an alternative approach to the use of antibiotics, Lydia Tabernero (University of Manchester, UK) proposed targeting a virulence factor from M. tuberculosis, MptpB, that is a secreted phosphatase critical in pathogenesis and survival of the bacilli in infected macrophages and animal models (Beresford et al., 2009). The use of a combination of structure-based and computational fragment screening permitted a new family of inhibitors to be designed with remarkable selectivity over human phosphatases and that impair bacillary growth inside macrophages. 


\section{Host-pathogen interactions I}

As stated previously, one of the main virulence features of M. tuberculosis is its capacity to manipulate phagosomal maturation and inhibit the biogenesis of bactericidal phagolysosomes. While $M$. tuberculosis was thought to exclusively reside within phagosomes inside host-cells, the team of Peter Peters (Netherland Cancer Institute, Amsterdam, The Netherlands) has demonstrated, using electron microscopy, that $M$. tuberculosis, and more generally pathogenic mycobacteria, can be visualized as free bacilli inside the host cell's cytosol (van der Wel et al., 2007). Translocation from the phagosome to the cytosol of the host cell was shown to be dependent on the ESX-1 type VII secretion system, as its introduction to the attenuated vaccine strain $M$. bovis $\mathrm{BCG}$, that naturally lacks ESX-1, is needed to trigger translocation. Among ESX-1 secreted proteins, the $6 \mathrm{kDa}$ Early Secreted Antigenic Target (ESAT-6) seems to play a main role in this process, as a mutation in the C-terminus of this protein impairs translocation (Houben et al., 2012a,b). The overall molecular organization of the ESX-1 system in in vitro cultivated $M$. tuberculosis was determined by immuno-precipitation techniques followed by 3-D modelling of the ESX-1 proteins EspB and EccA. In this context, Roxane Simeone (Institut Pasteur, Paris, France) presented recently published data where, using single-cell FRET imaging and automated fluorescence microscopy, mycobacterial translocation was quantified and the role of ESX-1 secretion system confirmed (Simeone et al., 2012).

David Russell (Cornell University, Ithaca, USA) reported complementary approaches to probe the micro-environment in which mycobacteria reside during infection, and to exploit this information in order to identify new antituberculous drugs. Using recombinant $M$. tuberculosis strains, in which GFP is expressed under the control of selected promoters responding to well defined stimuli, such as the $\operatorname{apr} A B C$ promoter (Abramovitch et al., 2011), direct temporal and spatial observation of M. tuberculosis acidic resistance or cholesterol utilization was possible in mouse tissues. A beautiful example of such probing of the host-pathogen interplay was provided using M. tuberculosis strains where GFP was expressed under the control of the hypoxia- and NO-induced $h s p X$ promoter. Strikingly, fluorescence of the strain was greatly decreased in KO mice impaired in NO production. Using a high-throughput technology based on automated confocal microscopy, a library of chemical compounds was screened in order to identify compounds able to selectively kill bacteria inside macrophages. This method led to the identification of a hundred promising hits, some of which were exclusively active against intracellular M. tuberculosis.

As a complement to the search for new drugs, Anna Coussens (NIMR, London, UK and University of Cape Town, South Africa) presented a fascinating study where molecules were searched for that could potentiate the well-established protective effect of Vitamin D3 against TB. Sodium 4phenylbutyrate (PBA) was shown to synergise with Vitamin D3 and kill intracellular $M$. tuberculosis through increasing the expression of antimicrobial peptides cathelicidin and lactoferrin. Moreover, PBA was found to significantly restrict $M$. tuberculosis growth in axenic conditions, thereby exhibiting a double level of antibacterial activity. A combination of vitamin D and PBA was proposed as an adjunct to current treatment as means of shortening antituberculosis therapy (Coussens et al., 2012).

\section{Host-pathogen interactions II}

Host-pathogen interaction is a finely balanced equilibrium between host defence mechanisms and bacterial survival strategies. Olivier Neyrolles (IPBS, Toulouse, France) illustrated the role of zinc intoxication in macrophages on the outcome of M. tuberculosis infection. Following M. tuberculosis phagocytosis, an intracellular pool of free zinc was shown to localise to the M. tuberculosis phagosome inside primary human macrophages. As high concentrations of zinc are toxic to $M$. tuberculosis, the identification of a bacterial detoxification system was sought. The P-ATPase CtpC was proposed to contribute to zinc detoxification through zinc efflux, as a mutant in the $c t p C$ gene was more sensitive to zinc in vitro, and was impaired in multiplication inside macrophages. Zinc accumulation in vacuoles is not restricted to $M$. tuberculosis as it is also observed in Escherichia coli and latex bead phagolysosomes. Together with similar mechanisms described for copper (Festa et al., 2011; Ward et al., 2010; Wolschendorf et al., 2011), this work reveals a new and unsuspected role for transition metals in innate defence against microbes (Botella et al., 2011) (Fig. 2).

Dictyostelium is a practical model to study host-pathogen interactions, as this unicellular organism is easily tractable at the genetic level. Taking Mycobacterium marinum as a model pathogen, Monica Hagedorn (Bernhard Nocht Institute, Hamburg, Germany) could show the existence of a mechanism for nonlytic ejection of cytosolic mycobacteria that was responsible for cell-to-cell bacterial transmission. This mechanism is mediated by the formation of a specific and short-lived actin structure called an 'ejectosome' at the plasma membrane of infected cells. In a Dictyostelium strain lacking $\mathrm{RacH}$, a small GTPase involved in actin cytoskeleton regulation, no ejectosomes were detected and mycobacterial cellto-cell spread was drastically reduced. Ejectosome formation appears to be dependent on the expression of the ESX-1 secretion system as no ejectosomes were observed in an $M$. marinum mutant lacking ESX-1 (Hagedorn et al., 2009).

While the major role of type II interferon (IFN- $\gamma$ ) in immune control of M. tuberculosis is well established, the role of type I IFN remained incompletely understood until recently. To address this, Ludovic Desvignes (NYU School of Medicine, New York, USA) explored the specific role of type I IFN in the outcome of $M$. tuberculosis infection in vivo. Using mice carrying null mutations in the type I IFNR, the type II IFNR, or both, the authors could demonstrate that in a type II IFNR-null background, the absence of type I IFN increases lung inflammation and causes earlier death of the animals following M. tuberculosis infection. While both IFNs are 


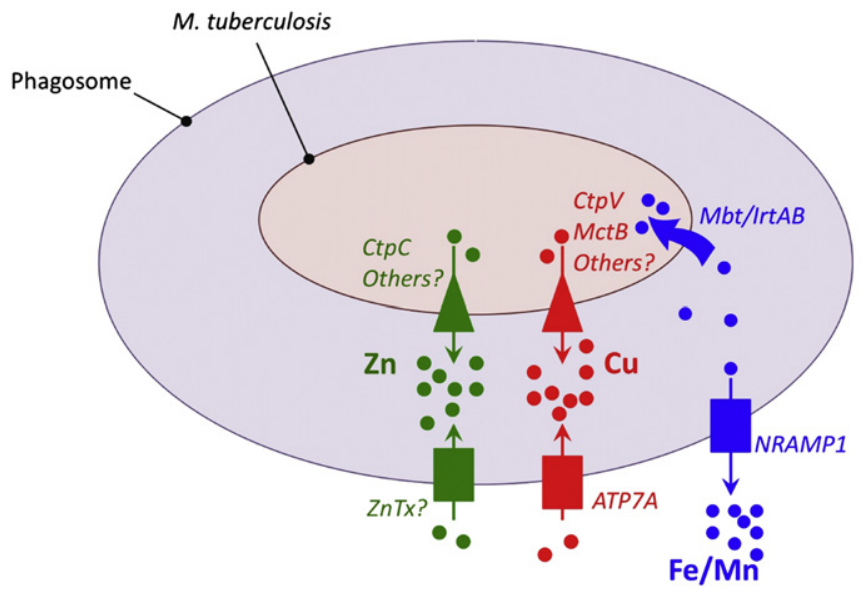

Fig. 2. Metallobiology of the mycobacterial phagosome. Within macrophages, M. tuberculosis is present in the phagosome, which is depleted of iron and possibly manganese by NRAMP1 and accumulates copper and zinc through the action of the copper transporter ATP7A and other as yet unidentified transporters that may belong to the ZnT/SLC30A family of zinc transporters. In response to iron limitation, $M$. tuberculosis produces iron uptake molecules, such as mycobactins (mbt) and IrtAB. In response to poisoning with copper and zinc, M. tuberculosis produces the copper efflux molecules CtpV and $\mathrm{MctB}$, and the putative zinc efflux pump CtpC. Due to a likely high level of redundancy among the mycobacterial Ctp transporters, it is possible that other members of this family of P-ATPases are involved in copper and zinc efflux. See (Botella et al., 2012) for a review. (Figure courtesy of Olivier Neyrolles, IPBS, Toulouse, France).

required for optimal recruitment of NK and myeloid cells to the site of infection, the lack of type I IFN specifically increases the number of infected macrophages in the lungs. This study confirmed the major role of type II IFN in restricting mycobacterial growth and revealed a significant function for type I IFN in limiting infectious site expansion (Desvignes et al., 2012).

Using the zebra fish and $M$. marinum as a host-pathogen model system, Lalita Ramakrishnan (University of Washington, Seattle, USA) presented data showing the positive role of the granuloma in mycobacterial colonisation of the host. Through secretion of ESAT-6, M. marinum can induce the expression of matrix metalloproteinase-9 (MMP-9) by epithelial cells, which in turn enhances macrophage recruitment (Volkman et al., 2010). The recently reported role of the lta4h locus, coding for a leukotriene hydrolase, in balancing host defence $v s$. inflammation-mediated tissue injury was discussed, with genetic-associated treatment of TB patients proposed (Tobin et al., 2010).

\section{Animal models and beyond}

There is an obvious need for appropriate and robust animal models in which to investigate the fundamental interactions between M. tuberculosis and its host, as well as providing the means for testing the efficacy of new drugs and vaccines. Jacques Grosset (John Hopkins University, USA \& KwaZuluNatal Research Institute, ZA) explored this theme by investigating the mode of action of pyrazinamide, a key sterilizing drug in the treatment of $\mathrm{TB}$, and its proposed immune- modulatory mechanism of action (Mendez et al., 2009). Experiments were conducted in immune-competent BALB/c and in athymic nude mice using both M. tuberculosis and $M$. bovis (the latter strain being naturally resistant to pyrazinamide). In these models the results were not conclusively able to rule in or rule out an immune-modulatory activity for pyrazinamide, suggesting that further work is needed to clarify this question.

M. tuberculosis infection results in a spectrum of granuloma types, but the mechanisms underlying granuloma formation and maintenance are unclear. Joanne Flynn (University of Pittsburgh, USA) took advantage of PET/CT imaging to study granuloma dynamics in macaques infected with $M$. tuberculosis. In particular, she showed that each granuloma starts with a single bacterium and has a unique trajectory in terms of size and inflammatory changes over time. Interestingly, the percentage of $\mathrm{T}$ cells within the granuloma that produce cytokines in response to mycobacterial antigen is very low, with only alternatively activated macrophages found throughout the granuloma, whereas classically activated macrophages are found only around the caesum.

Ludovic Tailleux (Institut Pasteur, Paris, France) has been deciphering how M. tuberculosis colonizes extrapulmonary sites. He found that upon M. tuberculosis infection, human macrophages express numerous factors including VEGF that controls the formation of new blood vessels. Inhibiting angiogenesis strongly decreases the spread of bacteria in murine models of the disease. This unexpected role of macrophages in the dissemination of the bacillus may explain many extrapulmonary forms of TB.

Stéphanie Boisson-Dupuis (The Rockfeller University, New York, USA) defined how genetic predisposition could explain the inter-individual variability to TB susceptibility. As an example, using a candidate gene approach in a cohort of paediatric patients with acute, disseminated TB, she found that 2 patients out of 50 had autosomal recessive complete IL12RB1 deficiency (Boisson-Dupuis et al., 2011). This finding, and others, goes to show that the outcome of TB infection is a complex interaction that depends both on the genotype of the host as well as that of the infecting M. tuberculosis strain.

\section{Concluding remarks}

The need for a deeper understanding of the immune response to infection is essential if we are to understand latent and active disease, and to provide markers for improved diagnosis, treatment monitoring, etc. Towards this goal, Anne O'Garra (NIMR, Mill Hill, London) has used whole blood transcriptomics to identify a transcript signature that differentiates active TB from both latent TB and other chronic inflammatory conditions. The transcript profile was dominated by neutrophil-driven IFN- $\gamma$ and type I IFN- $\alpha \beta$ signalling, revealing a key role for type I IFN signalling in TB pathogenesis (Berry et al., 2010).

Clif Barry (NIH, Bethesda, USA) then posed the question of how well 'lab-based' predictions of $M$. tuberculosis and its interaction with the host actually translate into what is seen in 
the clinic. One such prediction was based on the premise that since $M$. tuberculosis persists in vivo in a hypoxic environment, drugs acting under such conditions, such as metronizidaole, would prove effective in treating latent TB, and this held true in animal models (Lin et al., 2012a,b). However, clinical trials showed that metronizidole had limited efficacy for preventing reactivation TB. The converse was illustrated by linezolid, an oxazolidinone, which had poor efficacy in mouse models of TB. However, when given to a cohort of XDR-TB patients in addition to their standard drug regimen, linezolid treatment converted a significant portion of the patients to culture negativity, albeit with side effects linked to drug toxicity (Lee et al., 2012). This presentation, entitled 'an inconvenient truth from the clinic' begged the question: just how good are our current models of TB? Perhaps the answer to this question will be found at the next TB meeting in Paris; we look forward to it.

\section{Acknowledgements}

We would like to thank all our colleagues who agreed to share their unpublished data for this meeting report. We are also grateful to the meeting organisers for putting together such a thought-provoking and entertaining meeting.

\section{References}

Aagaard, C., Hoang, T., Dietrich, J., Cardona, P.J., Izzo, A., Dolganov, G., Schoolnik, G.K., Cassidy, J.P., et al., 2011. A multistage tuberculosis vaccine that confers efficient protection before and after exposure. Nat. Med. 17, 189-194.

Abramovitch, R.B., Rohde, K.H., Hsu, F.F., Russell, D.G., 2011. aprABC: a Mycobacterium tuberculosis complex-specific locus that modulates $\mathrm{pH}$ driven adaptation to the macrophage phagosome. Mol. Microbiol. 80, 678-694.

Arabolaza, A., D’Angelo, M., Comba, S., Gramajo, H., 2010. FasR, a novel class of transcriptional regulator, governs the activation of fatty acid biosynthesis genes in Streptomyces coelicolor. Mol. Microbiol. 78, 47-63.

Arnvig, K., Young, D., 2012. Non-coding RNA and its potential role in Mycobacterium tuberculosis pathogenesis. RNA Biol. 9, 427-436.

Arnvig, K.B., Comas, I., Thomson, N.R., Houghton, J., Boshoff, H.I., Croucher, N.J., Rose, G., Perkins, T.T., et al., 2011. Sequence-based analysis uncovers an abundance of non-coding RNA in the total transcriptome of Mycobacterium tuberculosis. PLoS Pathog. 7, e1002342.

Arnvig, K.B., Young, D.B., 2009. Identification of small RNAs in Mycobacterium tuberculosis. Mol. Microbiol. 73, 397-408.

Beresford, N.J., Mulhearn, D., Szczepankiewicz, B., Liu, G., Johnson, M.E., Fordham-Skelton, A., Abad-Zapatero, C., Cavet, J.S., et al., 2009. Inhibition of MptpB phosphatase from Mycobacterium tuberculosis impairs mycobacterial survival in macrophages. J. Antimicrob. Chemother. 63, 928-936.

Berry, M.P., Graham, C.M., McNab, F.W., Xu, Z., Bloch, S.A., Oni, T., Wilkinson, K.A., Banchereau, R., et al., 2010. An interferon-inducible neutrophil-driven blood transcriptional signature in human tuberculosis. Nature 466, 973-977.

Bigger, J.W., 1944. Treatment of staphylococcal infections with penicillin. Lancet 244, 497-500.

Bitter, W., Houben, E.N., Bottai, D., Brodin, P., Brown, E.J., Cox, J.S., Derbyshire, K., Fortune, S.M., et al., 2009. Systematic genetic nomenclature for type VII secretion systems. PLoS Pathog. 5, e1000507.

Blasco, B., Chen, J.M., Hartkoorn, R., Sala, C., Uplekar, S., Rougemont, J., Pojer, F., Cole, S.T., 2012. Virulence regulator EspR of Mycobacterium tuberculosis is a nucleoid-associated protein. PLoS Pathog. 8, e1002621.
Boisson-Dupuis, S., El Baghdadi, J., Parvaneh, N., Bousfiha, A., Bustamante, J., Feinberg, J., Samarina, A., Grant, A.V., et al., 2011. IL12Rbeta1 deficiency in two of fifty children with severe tuberculosis from Iran, Morocco, and Turkey. PLoS One 6, e18524.

Botella, H., Peyron, P., Levillain, F., Poincloux, R., Poquet, Y., Brandli, I., Wang, C., Tailleux, L., et al., 2011. Mycobacterial p(1)-type ATPases mediate resistance to zinc poisoning in human macrophages. Cell Host Microbe 10, 248-259.

Botella, H., Stadthagen, G., Lugo-Villarino, G., de Chastellier, C., Neyrolles, O., 2012. Metallobiology of host-pathogen interactions: an intoxicating new insight. Trends Microbiol. 20, 106-112.

Bottai, D., Di Luca, M., Majlessi, L., Frigui, W., Simeone, R., Sayes, F., Bitter, W., Brennan, M.J., et al., 2012. Disruption of the ESX-5 system of Mycobacterium tuberculosis causes loss of PPE protein secretion, reduction of cell wall integrity and strong attenuation. Mol. Microbiol. 83, 1195-1209.

Bottger, E.C., 2011. The ins and outs of Mycobacterium tuberculosis drug susceptibility testing. Clin. Microbiol. Infect. 17, 1128-1134.

Brisse, S., Supply, P., Brosch, R., Vincent, V., Gutierrez, M.C., 2006. "A reevaluation of M. prototuberculosis": continuing the debate. PLoS Pathog. 2, e95.

Christophe, T., Ewann, F., Jeon, H.K., Cechetto, J., Brodin, P., 2010. Highcontent imaging of Mycobacterium tuberculosis-infected macrophages: an in vitro model for tuberculosis drug discovery. Future Med. Chem. 2, 1283-1293.

Corbiere, V., Pottier, G., Bonkain, F., Schepers, K., Verscheure, V., Lecher, S., Doherty, T.M., Locht, C., et al., 2012. Risk stratification of latent tuberculosis defined by combined interferon gamma release assays. PLoS One 7, e43285.

Coussens, A.K., Wilkinson, R.J., Hanifa, Y., Nikolayevskyy, V., Elkington, P.T., Islam, K., Timms, P.M., Venton, T.R., et al., 2012. Vitamin $\mathrm{D}$ accelerates resolution of inflammatory responses during tuberculosis treatment. Proc. Natl. Acad. Sci. U. S. A. 109, 15449-15454.

de Carvalho, L.P., Zhao, H., Dickinson, C.E., Arango, N.M., Lima, C.D., Fischer, S.M., Ouerfelli, O., Nathan, C., et al., 2010. Activity-based metabolomic profiling of enzymatic function: identification of Rv1248c as a mycobacterial 2-hydroxy-3-oxoadipate synthase. Chem. Biol. 17, 323-332.

Desvignes, L., Wolf, A.J., Ernst, J.D., 2012. Dynamic roles of type I and type II IFNs in early infection with Mycobacterium tuberculosis. J. Immunol. 188, 6205-6215.

Enarson, D., 2004. TB and IUATLD. Pediatr. Pulmonol. Suppl. 26, 55-56.

Festa, R.A., Jones, M.B., Butler-Wu, S., Sinsimer, D., Gerads, R., Bishai, W.R., Peterson, S.N., Darwin, K.H., 2011. A novel copperresponsive regulon in Mycobacterium tuberculosis. Mol. Microbiol. 79, $133-148$.

Gold, B., Pingle, M., Brickner, S.J., Shah, N., Roberts, J., Rundell, M., Bracken, W.C., Warrier, T., et al., 2012. Nonsteroidal anti-inflammatory drug sensitizes Mycobacterium tuberculosis to endogenous and exogenous antimicrobials. Proc. Natl. Acad. Sci. U. S. A. 109, 16004-16011.

Grode, L., Seiler, P., Baumann, S., Hess, J., Brinkmann, V., Nasser Eddine, A., Mann, P., Goosmann, C., et al., 2005. Increased vaccine efficacy against tuberculosis of recombinant Mycobacterium bovis bacille Calmette-Guerin mutants that secrete listeriolysin. J. Clin. Invest. 115, 2472-2479.

Grzegorzewicz, A.E., Kordulakova, J., Jones, V., Born, S.E., Belardinelli, J.M., Vaquie, A., Gundi, V.A., Madacki, J., et al., 2012a. A common mechanism of inhibition of the Mycobacterium tuberculosis mycolic acid biosynthetic pathway by isoxyl and thiacetazone. J. Biol. Chem. 287, 38434-38441.

Grzegorzewicz, A.E., Pham, H., Gundi, V.A., Scherman, M.S., North, E.J., Hess, T., Jones, V., Gruppo, V., et al., 2012b. Inhibition of mycolic acid transport across the Mycobacterium tuberculosis plasma membrane. Nat. Chem. Biol. 8, 334-341.

Gutierrez, M.C., Brisse, S., Brosch, R., Fabre, M., Omais, B., Marmiesse, M., Supply, P., Vincent, V., 2005. Ancient origin and gene mosaicism of the progenitor of Mycobacterium tuberculosis. PLoS Pathog. 1, e5.

Guyer, D.M., Henderson, I.R., Nataro, J.P., Mobley, H.L., 2000. Identification of sat, an autotransporter toxin produced by uropathogenic Escherichia coli. Mol. Microbiol. 38, 53-66. 
Hagedorn, M., Rohde, K.H., Russell, D.G., Soldati, T., 2009. Infection by tubercular mycobacteria is spread by nonlytic ejection from their amoeba hosts. Science 323, 1729-1733.

Hoffmann, C., Leis, A., Niederweis, M., Plitzko, J.M., Engelhardt, H., 2008. Disclosure of the mycobacterial outer membrane: cryo-electron tomography and vitreous sections reveal the lipid bilayer structure. Proc. Natl. Acad. Sci. U. S. A. 105, 3963-3967.

Homolka, S., Niemann, S., Russell, D.G., Rohde, K.H., 2010. Functional genetic diversity among Mycobacterium tuberculosis complex clinical isolates: delineation of conserved core and lineage-specific transcriptomes during intracellular survival. PLoS Pathog. 6, e1000988.

Houben, D., Demangel, C., van Ingen, J., Perez, J., Baldeon, L., Abdallah, A.M., Caleechurn, L., Bottai, D., et al., 2012a. ESX-1-mediated translocation to the cytosol controls virulence of mycobacteria. Cell. Microbiol. 14, 1287-1298.

Houben, E.N., Bestebroer, J., Ummels, R., Wilson, L., Piersma, S.R., Jimenez, C.R., Ottenhoff, T.H., Luirink, J., et al., 2012b. Composition of the type VII secretion system membrane complex. Mol. Microbiol. 86, $472-484$.

Hougardy, J.M., Schepers, K., Place, S., Drowart, A., Lechevin, V., Verscheure, V., Debrie, A.S., Doherty, T.M., et al., 2007. Heparin-bindinghemagglutinin-induced IFN-gamma release as a diagnostic tool for latent tuberculosis. PLoS One 2, e926.

Kumar, A., Zhang, M., Zhu, L., Liao, R.P., Mutai, C., Hafsat, S., Sherman, D.R., Wang, M.W., 2012. High-throughput screening and sensitized bacteria identify an M. tuberculosis dihydrofolate reductase inhibitor with whole cell activity. PLoS One 7, e39961.

La Rosa, V., Poce, G., Canseco, J.O., Buroni, S., Pasca, M.R., Biava, M., Raju, R.M., Porretta, G.C., et al., 2012. MmpL3 is the cellular target of the antitubercular pyrrole derivative BM212. Antimicrob. Agents Chemother. $56,324-331$.

Layre, E., Sweet, L., Hong, S., Madigan, C.A., Desjardins, D., Young, D.C., Cheng, T.Y., Annand, J.W., et al., 2011. A comparative lipidomics platform for chemotaxonomic analysis of Mycobacterium tuberculosis. Chem. Biol. $18,1537-1549$

Lee, M., Lee, J., Carroll, M.W., Choi, H., Min, S., Song, T., Via, L.E., Goldfeder, L.C., et al., 2012. Linezolid for treatment of chronic extensively drug-resistant tuberculosis. N. Engl. J. Med. 367, 1508-1518.

Lin, P.L., Dartois, V., Johnston, P.J., Janssen, C., Via, L., Goodwin, M.B., Klein, E., Barry 3rd, C.E., et al., 2012a. Metronidazole prevents reactivation of latent Mycobacterium tuberculosis infection in macaques. Proc. Natl. Acad. Sci. U. S. A. 109, 14188-14193.

Lin, P.L., Dietrich, J., Tan, E., Abalos, R.M., Burgos, J., Bigbee, C., Bigbee, M., Milk, L., et al., 2012b. The multistage vaccine H56 boosts the effects of BCG to protect cynomolgus macaques against active tuberculosis and reactivation of latent Mycobacterium tuberculosis infection. J. Clin. Invest. 122, 303-314.

Maertzdorf, J., Weiner 3rd, J., Mollenkopf, H.J., Bauer, T., Prasse, A., MullerQuernheim, J., Kaufmann, S.H., 2012. Common patterns and diseaserelated signatures in tuberculosis and sarcoidosis. Proc. Natl. Acad. Sci. U. S. A. $109,7853-7858$.

Makarov, V., Manina, G., Mikusova, K., Mollmann, U., Ryabova, O., SaintJoanis, B., Dhar, N., Pasca, M.R., et al., 2009. Benzothiazinones kill Mycobacterium tuberculosis by blocking arabinan synthesis. Science 324, 801-804.

Mendez, S., Traslavina, R., Hinchman, M., Huang, L., Green, P., Cynamon, M.H., Welch, J.T., 2009. The antituberculosis drug pyrazinamide affects the course of cutaneous leishmaniasis in vivo and increases activation of macrophages and dendritic cells. Antimicrob. Agents Chemother. 53, 5114-5121.

Mishra, B.B., Rathinam, V.A., Martens, G.W., Martinot, A.J., Kornfeld, H., Fitzgerald, K.A., Sassetti, C.M., 2013. Nitric oxide controls the immunopathology of tuberculosis by inhibiting NLRP3 inflammasomedependent processing of IL-1beta. Nat. Immunol. 14, 52-60.

Molle, V., Kremer, L., 2010. Division and cell envelope regulation by Ser/Thr phosphorylation: Mycobacterium shows the way. Mol. Microbiol. 75, $1064-1077$
Nambiar, J.K., Pinto, R., Aguilo, J.I., Takatsu, K., Martin, C., Britton, W.J., Triccas, J.A., 2012. Protective immunity afforded by attenuated, PhoPdeficient Mycobacterium tuberculosis is associated with sustained generation of CD4+ T-cell memory. Eur. J. Immunol. 42, 385-392.

Niederweis, M., Danilchanka, O., Huff, J., Hoffmann, C., Engelhardt, H., 2010. Mycobacterial outer membranes: in search of proteins. Trends Microbiol. 18, 109-116.

O’Hare, H.M., Duran, R., Cervenansky, C., Bellinzoni, M., Wehenkel, A.M., Pritsch, O., Obal, G., Baumgartner, J., et al., 2008. Regulation of glutamate metabolism by protein kinases in mycobacteria. Mol. Microbiol. 70, $1408-1423$.

Pathan, A.A., Minassian, A.M., Sander, C.R., Rowland, R., Porter, D.W., Poulton, I.D., Hill, A.V., Fletcher, H.A., et al., 2012. Effect of vaccine dose on the safety and immunogenicity of a candidate TB vaccine, MVA85A, in BCG vaccinated UK adults. Vaccine 30, 5616-5624.

Pethe, K., Alonso, S., Biet, F., Delogu, G., Brennan, M.J., Locht, C., Menozzi, F.D., 2001. The heparin-binding haemagglutinin of M. tuberculosis is required for extrapulmonary dissemination. Nature 412, 190-194.

Picotti, P., Aebersold, R., 2012. Selected reaction monitoring-based proteomics: workflows, potential, pitfalls and future directions. Nat. Methods 9, $555-566$

Salzman, V., Mondino, S., Sala, C., Cole, S.T., Gago, G., Gramajo, H., 2010. Transcriptional regulation of lipid homeostasis in mycobacteria. Mol. Microbiol. 78, 64-77.

Sayes, F., Sun, L., Di Luca, M., Simeone, R., Degaiffier, N., Fiette, L., Esin, S., Brosch, R., et al., 2012. Strong immunogenicity and crossreactivity of Mycobacterium tuberculosis ESX-5 type VII secretion: encoded PE-PPE proteins predicts vaccine potential. Cell Host Microbe $11,352-363$.

Schnappinger, D., Ehrt, S., Voskuil, M.I., Liu, Y., Mangan, J.A., Monahan, I.M., Dolganov, G., Efron, B., et al., 2003. Transcriptional adaptation of Mycobacterium tuberculosis within macrophages: insights into the phagosomal environment. J. Exp. Med. 198, 693-704.

Scriba, T.J., Tameris, M., Smit, E., van der Merwe, L., Hughes, E.J., Kadira, B., Mauff, K., Moyo, S., et al., 2012. A phase IIa trial of the new tuberculosis vaccine, MVA85A, in HIV- and/or Mycobacterium tuberculosis-infected adults. Am. J. Respir. Crit. Care Med. 185, 769-778.

Shi, W., Zhang, X., Jiang, X., Yuan, H., Lee, J.S., Barry 3rd, C.E., Wang, H., Zhang, W., et al., 2011. Pyrazinamide inhibits trans-translation in Mycobacterium tuberculosis. Science 333, 1630-1632.

Simeone, R., Bobard, A., Lippmann, J., Bitter, W., Majlessi, L., Brosch, R., Enninga, J., 2012. Phagosomal rupture by Mycobacterium tuberculosis results in toxicity and host cell death. PLoS Pathog. 8, e1002507.

Stanley, S.A., Grant, S.S., Kawate, T., Iwase, N., Shimizu, M., Wivagg, C., Silvis, M., Kazyanskaya, E., et al., 2012. Identification of novel inhibitors of M. tuberculosis growth using whole cell based high-throughput screening. ACS Chem. Biol. 7, 1377-1384.

Subbian, S., Tsenova, L., O’Brien, P., Yang, G., Kushner, N.L., Parsons, S., Peixoto, B., Fallows, D., et al., 2012. Spontaneous latency in a rabbit model of pulmonary tuberculosis. Am. J. Pathol. 181, 1711-1724.

Tahlan, K., Wilson, R., Kastrinsky, D.B., Arora, K., Nair, V., Fischer, E., Barnes, S.W., Walker, J.R., et al., 2012. SQ109 targets MmpL3, a membrane transporter of trehalose monomycolate involved in mycolic acid donation to the cell wall core of Mycobacterium tuberculosis. Antimicrob. Agents Chemother. 56, 1797-1809.

Tobin, D.M., Vary Jr., J.C., Ray, J.P., Walsh, G.S., Dunstan, S.J., Bang, N.D., Hagge, D.A., Khadge, S., et al., 2010. The lta4h locus modulates susceptibility to mycobacterial infection in zebrafish and humans. Cell 140, 717-730.

Toledo-Arana, A., Repoila, F., Cossart, P., 2007. Small noncoding RNAs controlling pathogenesis. Curr. Opin. Microbiol. 10, 182-188.

van der Wel, N., Hava, D., Houben, D., Fluitsma, D., van Zon, M., Pierson, J., Brenner, M., Peters, P.J., 2007. M. tuberculosis and M. leprae translocate from the phagolysosome to the cytosol in myeloid cells. Cell 129, $1287-1298$. 
van Soolingen, D., Hoogenboezem, T., de Haas, P.E., Hermans, P.W., Koedam, M.A., Teppema, K.S., Brennan, P.J., Besra, G.S., et al., 1997. A novel pathogenic taxon of the Mycobacterium tuberculosis complex, Canetti: characterization of an exceptional isolate from Africa. Int. J. Syst. Bacteriol. 47, 1236-1245.

Volkman, H.E., Pozos, T.C., Zheng, J., Davis, J.M., Rawls, J.F., Ramakrishnan, L., 2010. Tuberculous granuloma induction via interaction of a bacterial secreted protein with host epithelium. Science 327, 466-469.

Wagner, T., Bellinzoni, M., Wehenkel, A., O’Hare, H.M., Alzari, P.M., 2011. Functional plasticity and allosteric regulation of alpha-ketoglutarate decarboxylase in central mycobacterial metabolism. Chem. Biol. 18, 1011-1020.

Ward, S.K., Abomoelak, B., Hoye, E.A., Steinberg, H., Talaat, A.M., 2010. CtpV: a putative copper exporter required for full virulence of Mycobacterium tuberculosis. Mol. Microbiol. 77, 1096-1110.

Watanabe, S., Zimmermann, M., Goodwin, M.B., Sauer, U., Barry 3rd, C.E., Boshoff, H.I., 2011. Fumarate reductase activity maintains an energized membrane in anaerobic Mycobacterium tuberculosis. PLoS Pathog. 7, e1002287.

Wolschendorf, F., Ackart, D., Shrestha, T.B., Hascall-Dove, L., Nolan, S., Lamichhane, G., Wang, Y., Bossmann, S.H., et al., 2011. Copper resistance is essential for virulence of Mycobacterium tuberculosis. Proc. Natl. Acad. Sci. U. S. A. 108, 1621-1626.

$\mathrm{Xu}$, H., Hegde, S.S., Blanchard, J.S., 2011. Reversible acetylation and inactivation of Mycobacterium tuberculosis acetyl-CoA synthetase is dependent on cAMP. Biochemistry 50, 5883-5892.
Alexandre Gouzy

Jérôme Nigou

Martine Gilleron

Olivier Neyrolles

Centre National de la Recherche Scientifique, Institut de Pharmacologie et de Biologie Structurale, Toulouse, France Université de Toulouse, Université Paul Sabatier, Institut de Pharmacologie et de Biologie Structurale, Toulouse, France

Ludovic Tailleux

Unit of Mycobacterial Genetics, Institut Pasteur, 75015 Paris,

France

Stephen V. Gordon*

UCD Schools of Veterinary Medicine, Medicine and Medical Science, and Biomolecular and Biomedical Science, University College Dublin, Dublin, Ireland

*Corresponding author. E-mail address: stephen.gordon@ucd.ie 